\title{
Modified hanging manoeuvre facilitates inferior vena cava resection and reconstruction during extended right hepatectomy: A technical case report
}

\author{
SHAMIR O.CAWICH ${ }^{1 *}$, DEXTER A.W. THOMAS ${ }^{1 *}$, VINDRA RAGOONANAN $^{1 *}$, \\ CHUNILAL RAMJIT $^{1 *}$, DYLAN NARINESINGH ${ }^{1 *}$, VIJAY NARAYNSINGH $^{1 *}$ and NEIL PEARCE ${ }^{2 *}$ \\ ${ }^{1}$ Department of Clinical Surgical Sciences, University of the West Indies, St. Augustine, The Republic of Trinidad and Tobago; \\ ${ }^{2}$ Hepatobiliary Division, Department of Surgery, Southampton General Hospital, SO16 6YD Southampton, UK
}

Received November 22, 2016; Accepted July 22, 2017

DOI: $10.3892 / \mathrm{mco} .2017 .1352$

\begin{abstract}
Liver resections are safe when performed by specialized hepatobiliary teams. However, complex liver resections are accompanied by significant perioperative risk and they may require modifications of the conventional surgical techniques. We herein report the case of a 54-year-old male patient who underwent an extended right liver resection with en bloc resection and reconstruction of the inferior vena cava. For this complex resection, a modification of the standard operative technique was required. A modified hanging manoeuvre was performed using two 19Fr nasogastric tubes outside the traditional avascular plane to facilitate resection. This modification of the hanging manoeuvre was proven to be feasible and safe, and it is recommended for inclusion in the armamentarium of hepatobiliary surgeons when complex resections are required.
\end{abstract}

\section{Introduction}

Liver resections are feasible and safe procedures when performed by specialized hepatobiliary teams (1). However, in certain, more complex cases, applying techniques such as the liver hanging manoeuvre may be required (2). In addition, when there is compression or malignant invasion of the inferior vena cava (IVC) by hepatic tumours, as indicated by preoperative radiological imaging $(3,4)$, the associated perioperative morbidity and mortality increase. In order to mitigate these risks and safely perform complex resections, modifications of the conventional liver resection techniques become necessary. We herein report the technical modifications of the

Correspondence to: Dr Shamir O. Cawich, Department of Clinical Surgical Sciences, University of the West Indies, St. Augustine Campus, St. Augustine, The Republic of Trinidad and Tobago

E-mail: socawich@hotmail.com

*Contributed equally

Key words: liver, hepatectomy, inferior vena cava, reconstruction, resection, hanging manoeuvre classic hanging manoeuvre used in a patient with colorectal liver metastasis (CRLM) who required an extended right hepatectomy with en bloc resection and reconstruction of the IVC.

\section{Case report}

A 54-year old man presented 3 years after right hemicolectomy for a caecal carcinoma with an asymptomatic CRLM in the right liver. The patient had completed 8 cycles of adjuvant systemic treatment with leucovorin, flourouracil and oxaliplatin (FOLFOX regimen) after the index right hemicolectomy. After CRLM was detected on surveillance computed tomography (CT) scans, 3 courses of second-line bevacizumab-based systemic therapy were administered. There was minimal demonstrable response, so the patient was referred to the hepatobiliary team for evaluation.

On CT scans, a solitary 25.4-cm CRLM was identified in the right hemi-liver extending into $\mathrm{S}_{\mathrm{IV}}$. There was suspected caval invasion on CT scans, as evidenced by distortion of the upper IVC and loss of the plane at the posterior liver surface (Figs. 1 and 2). Although the CRLM completely encased the right hepatic vein and appeared to involve the middle hepatic vein, the left hepatic vein remained uninvolved and the hilar structures were tumour-free.

A decision was made to proceed with extended right hepatectomy, with planned resection and reconstruction of the IVC. The abdomen was accessed using an upper midline incision with transverse extension. The line of parenchymal transection was identified using intraoperative ultrasound to select a plane that was clear of the margins of the tumour. The CRLM occupied the entire right upper quadrant (Fig. 3), effectively precluding visualization of diaphragmatic attachments. Without being able to mobilize the right liver for conventional resection, transection via the anterior approach without prior mobilization was considered, which would require a hanging manoeuvre to be executed safely. The classic hanging manoeuvre involves lifting the liver with a tape passed along the avascular plane at the 10-11 o'clock position over the IVC (2). In this case, preoperative scans suggested that this avascular plane was involved by the tumour. Therefore, blind passage of an instrument in this plane would be likely to cause 
torrential bleeding and breach the tumour, preventing R0 resection.

Instead, a modification of the classic hanging manoeuvre was used. Initially, the left hepatic vein junction was dissected out superiorly to establish access. A DeBakey vascular clamp was then passed over the anterior surface of the retro-hepatic IVC at the 2-3 o'clock position, directing it cephalad to exit at the left side of the left hepatic vein. This facilitated passage of a 20Fr nasogastric tube posteromedially behind the liver. Traction on this nasogastric tube anteriorly and to the left allowed partially lifting the liver with the heavy CRLM off the IVC. This allowed passing a second DeBakey clamp over the IVC at the 1 o'clock position with less resistance. The second instrument was directed between the middle and left hepatic veins, and was used to pass a second nasogastric tube in that plane (Fig. 4). The cephalad end of the second nasogastric tube was tied to our Omnitract ${ }^{\circledR}$ retractor and the surgeon pulled on the caudal end to provide controlled traction (Fig. 5).

This allowed performing an anterior transection of the hepatic parenchyma at the line previously selected with intraoperative ultrasound, guided down to the uninvolved IVC surface with controlled traction on the nasogastric tube. Once anterior transection was complete, the point of tumour invasion into the IVC was visualized (Fig. 6). The anterior wall of the IVC was invaded by the tumour for $5-6 \mathrm{~cm}$ in length and $25 \%$ of its diameter. The IVC was controlled with side-biting Satinsky clamps and the IVC wall was resected en bloc with the tumour to achieve 3-5-mm margins. The IVC was reconstructed primarily with 4/0 PDS sutures, resulting in narrowing to approximately $2 / 3$ of its original diameter (Fig. 7).

After the parenchymal transection and IVC repair were completed, the operation proceeded in a conventional manner. The right liver was mobilized by dividing the right triangular and coronary ligaments under direct visualization. The right hepatic pedicle was dissected and ligated using 3/0 polypropylene sutures. The right liver was then removed from the abdomen.

The operation was completed uneventfully in $315 \mathrm{~min}$. The total blood loss was estimated to be $1,100 \mathrm{ml}$ and the patient required transfusion of two units of packed cells intraoperatively. The postoperative recovery was also uneventful. The patient remained in the high dependency unit for $48 \mathrm{~h}$. The remaining recovery was uneventful and he was discharged from the hospital on the tenth postoperative day.

Histopathological assessment of the specimen confirmed CRLM with malignant invasion of the IVC. The parenchymal resection margins were clear of tumour. The tumour had invaded into the IVC, but the caval resection margins were histologically clear (Fig. 8).

Written informed consent was obtained from the patient regarding the publication of this case report and accompanying images.

\section{Discussion}

Malignant invasion of the IVC is difficult to predict on preoperative imaging, as it is a low-pressure vessel that may easily be compressed by hepatic tumours $(3,4)$. The radiological criteria predictive of malignant invasion include: Longitudinal
IVC indentation for $>50 \mathrm{~mm}$, transverse compression of $>50 \%$ of the IVC circumference, lesions protruding into the IVC lumen, and the presence of well-developed collaterals (4). Maeba et al (4) reported that the presence of $\geq 1$ of these radiological criteria may predict malignant IVC invasion with $60 \%$ overall accuracy. Using these criteria, IVC invasion was anticipated in our patient, since there was compression of $>50 \%$ of the transverse IVC diameter and $>5 \mathrm{~cm}$ longitudinal IVC compression. Endoscopic ultrasonography may have added to the preoperative assessment of malignant IVC invasion (5), but it was unavailable.

Despite malignant IVC invasion, an aggressive attempt was made at R0 margin clearance, as IVC resection and reconstruction is currently considered to be potentially curative when it results in histologically clear margins $(3,4,6-8)$. In these cases, the conventional techniques for liver resection may not be feasible. The conventional technique involves complete mobilization of the right liver in order to approach the IVC and to control the hepato-caval junction prior to parenchymal transection (9). Mobilization was not possible in the present case, as the large CRLM precluded visualization and dissection of the right triangular and coronary ligaments. It would also have been ill-advised from an oncological standpoint, as there is a risk of tumour rupture and seeding with the force that would be applied to mobilize a fixed and non-compliant liver heavily infiltrated with tumour. Therefore, two techniques were utilized for resection: The hanging manoeuvre and anterior parenchymal transection.

Ozawa (10) described the anterior transection technique in 1990. This technique was popularized over the next decade (11-15), driven by the perceived disadvantages of conventional right liver mobilization, namely impaired hepatic blood flow with rotational displacement of the liver (10), potential avulsion of hepatic veins (13), iatrogenic tumour rupture $(13,16)$, and hematogenous dissemination of malignant cells when the hepatic veins remained patent (16-19).

Liu et al (13) were the first to evaluate this in a prospective randomized trial, in which 120 patients undergoing major resections were randomized to hepatectomy via the anterior or the conventional approach. The authors did not include any patients with IVC invasion, but there were caudate resections in 7 patients with conventional and in 4 patients with anterior transection. There were no attempts at the hanging manoeuvre in that study. There was a statistically significant reduction in the number of patients with massive blood loss $>21(8.3$ vs. $28.3 \% ; \mathrm{P}=0.005)$, reduced transfusion requirements $(0$ vs. $0.3 \mathrm{l} ; \mathrm{P}=0.001)$ and better median survival (68.1 vs. 22.6 months; $\mathrm{P}=0.006$ ) with the anterior vs. conventional transection technique, respectively. However, Capussotti et al (20) prospectively randomized 65 patients undergoing right hepatectomy via the conventional or anterior approach, excluding patients with caudate lobe involvement $(n=9)$ or IVC invasion $(n=3)$. Although the authors routinely attempted the hanging manoeuvre, it was only completed in $30 / 33$ patients in the anterior transection group, being abandoned due to adhesions $(n=2)$ or major bleeding $(n=1)$, and they could not demonstrate any significant advantage with the anterior vs. the conventional approach in terms of overall blood loss (437 vs. $500 \mathrm{ml}$, respectively; $\mathrm{P}=0.960)$, perioperative transfusion rates (18 vs. $9.3 \%$, respectively; $\mathrm{P}=0.253)$ or perioperative mortality $(\mathrm{P}=0.746)$. 


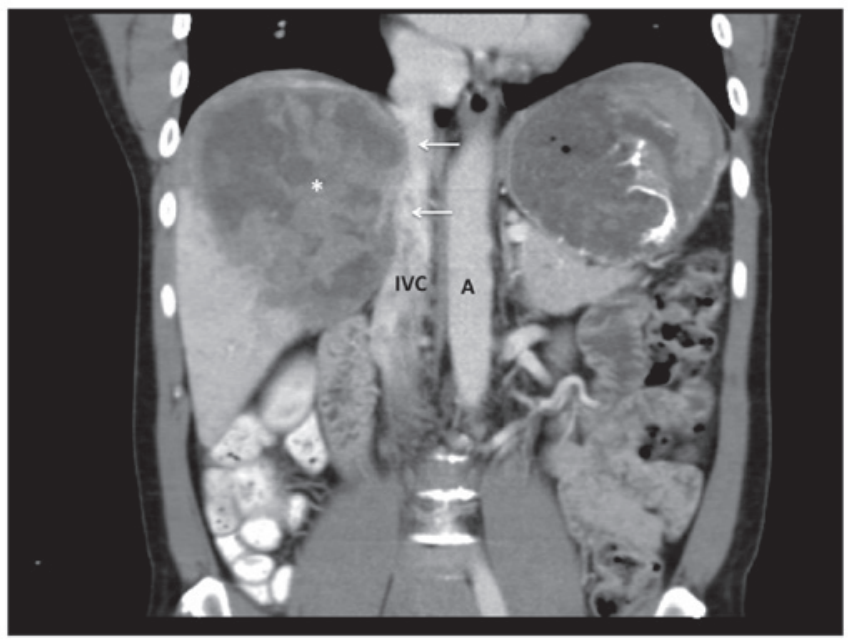

Figure 1. Coronal computed tomography scan showing a metastatic liver lesion (asterisk) occupying most of the right hemi-liver. The inferior vena cava (IVC) is indented by the metastatic deposit (arrows), particularly in its most cephalad portion, where there is loss of the plane between the tumour and the IVC. A, aorta.

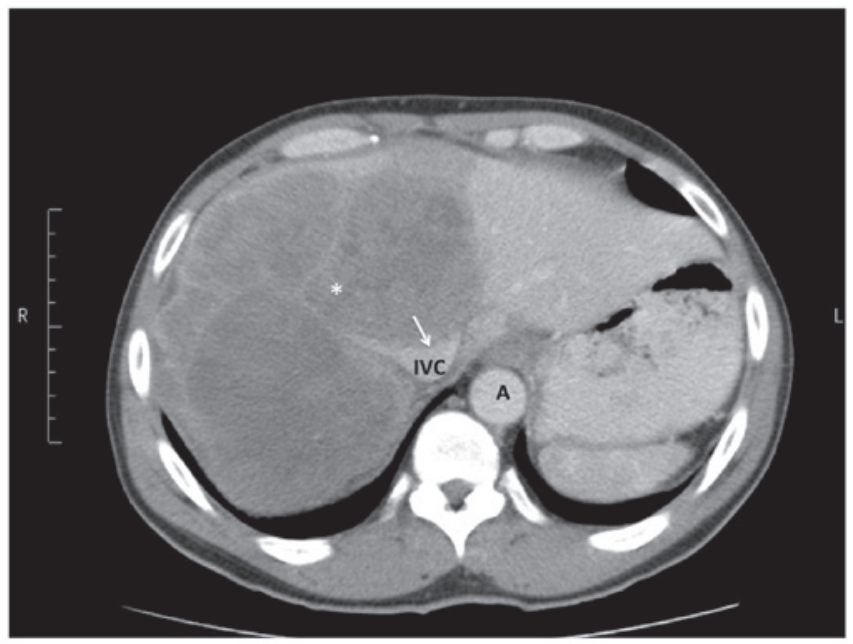

Figure 2. Axial computed tomography scan demonstrating the large tumour (asterisk) invading the upper portion of the inferior vena cava (IVC) as demonstrated by the irregular indentation of the anterior IVC wall (arrow). The right hepatic vein is completely encased in the tumour. A, aorta.

Thus, Capussotti et al (20) did not support routine use of the anterior approach.

Due to the conflicting data, Li et al (16) performed a meta-analysis of 807 patients across 2 prospective randomized trials $(13,20)$, plus an additional 6 non-randomized controlled trials $(11,12,21-24)$ comparing 444 patients undergoing conventional right hepatectomy to 363 undergoing right hepatectomy via anterior transection. The authors reported that the anterior approach resulted in significant reductions in transfusion rate ( 35 vs. $57.3 \%$; $\mathrm{P}<0.01)$, mortality $(1.9$ vs. $7.4 \%$; $\mathrm{P}<0.01)$ and local recurrence (48.9 vs. $62.9 \% ; \mathrm{P}<0.01$; odds ratio $=0.57 ; 95 \%$ confidence interval: 0.37-0.87). Although existing data lean towards the anterior approach, there are currently insufficient high-quality data to determine which is the optimal method for right hepatectomy. It is clear, however, that hepatobiliary surgeons should be familiar with the anterior transection tech-

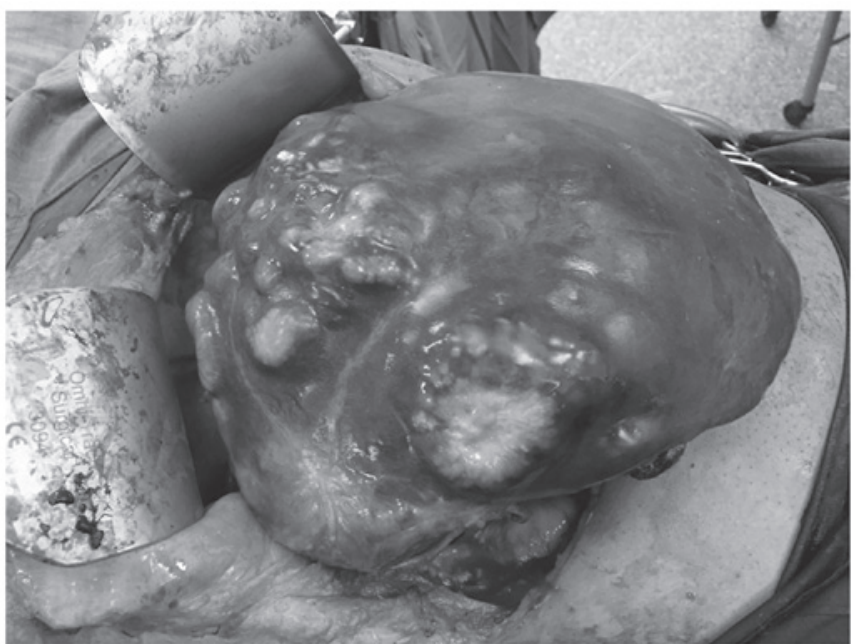

Figure 3. Intraoperative view of a metastatic liver lesion occupying the entire right lobe, making exposure difficult.

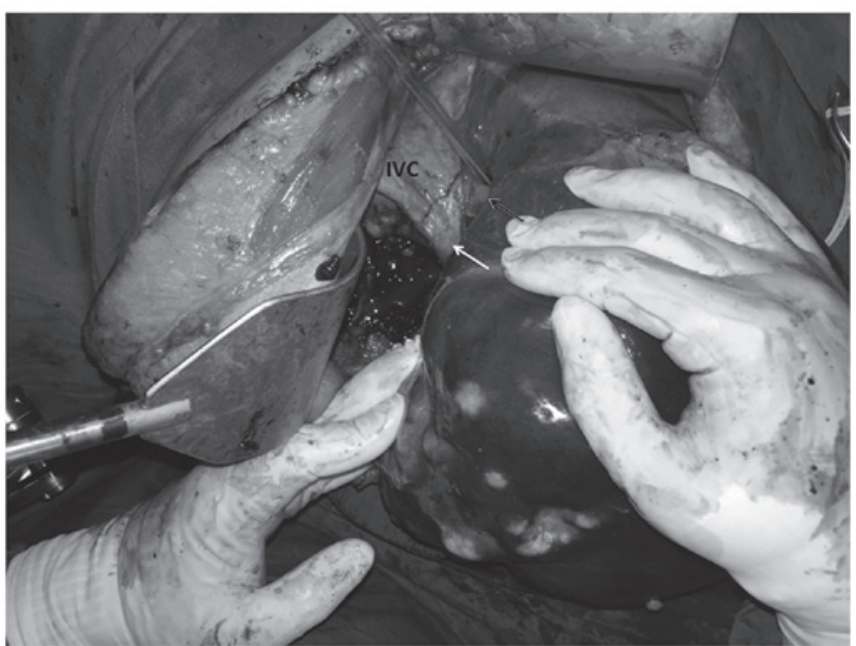

Figure 4. The second nasogastric tube is passed anterior to the inferior vena cava (IVC) and directed between the middle hepatic vein (yellow arrow) and the left hepatic vein (not visible due to overlying tumour) to the left of the tumour. White arrow, right hepatic vein.

nique as a part of their armamentarium. In the present case, the anterior transection technique was deemed necessary as i) the bulky right liver could not be mobilized by the conventional technique and ii) the point of malignant IVC invasion could be approached in a controlled manner in preparation for resection.

The hanging manoeuvre was also utilized in order to guide the line of transection and control bleeding from deep parenchymal vessels. Several investigators have reported good outcomes when they used the combination of anterior transection and the hanging manoeuvre to complete resections for a variety of pathologies $(11,13,25-29)$. Apart from serving as a directional guide during parenchymal transection (25), utilizing the hanging manoeuvre is associated with a lower risk of tumour dissemination into the hepatic veins (13), reduced incidence of tumour rupture $(11,13)$, reduced blood loss (28), lower operative time (28), reduced transfusion requirements (28), and shorter duration of hospitalization (26). 


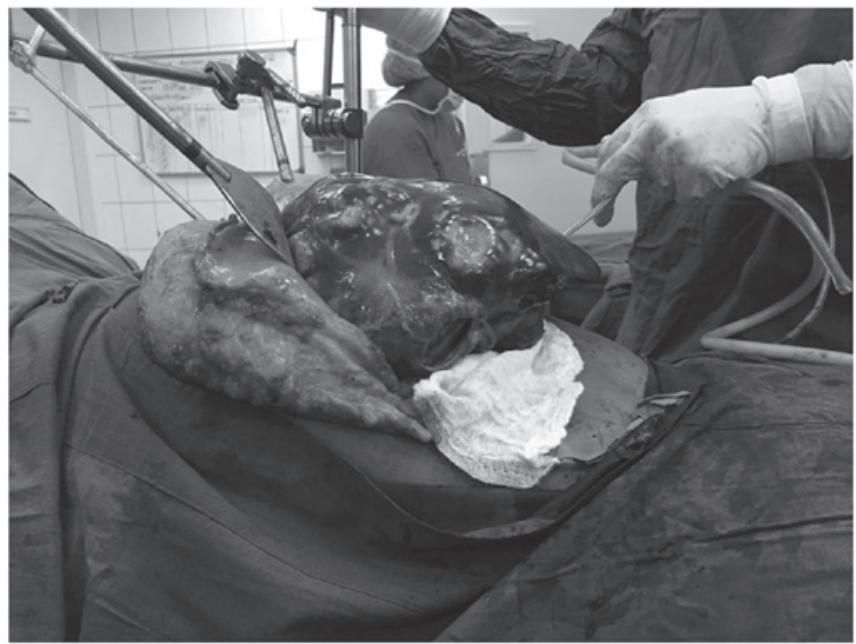

Figure 5. The completed hanging manoeuvre. The surgeon pulls on the second nasogastric tube in order to provide controlled traction to deliver the tumour for parenchymal transection.

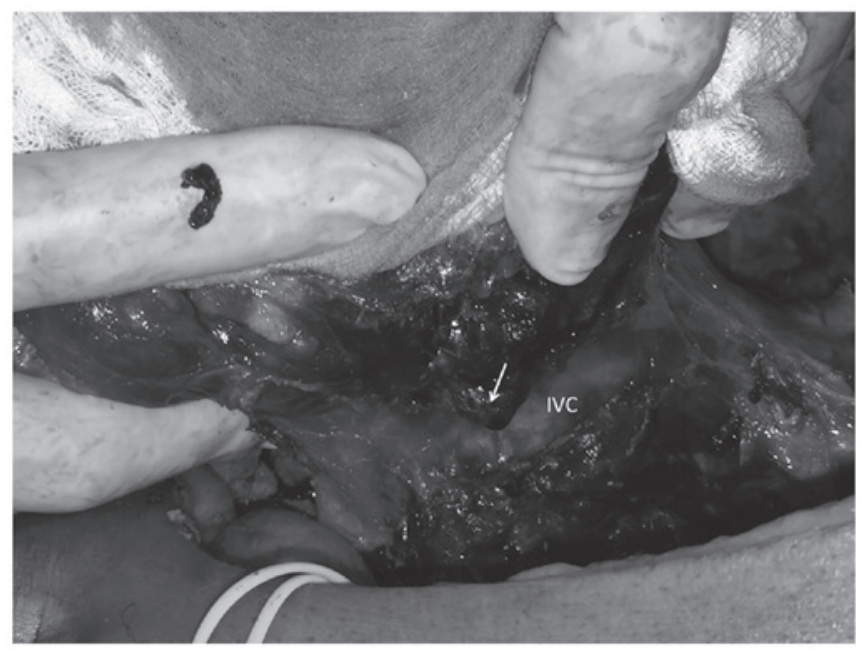

Figure 6. The point of tumour invasion into the inferior vena cava (IVC; arrow) is shown, as evidenced by tethering and angulation of the IVC with tumour manipulation.

Malignant IVC invasion was initially considered an absolute contraindication to the hanging manoeuvre (30). However, there have been published case reports $(29,31,25)$ and one small series of 7 cases (27), where this manoeuvre was used to perform complex resections facilitating partial IVC resection and reconstruction in patients with malignant invasion. There were good outcomes in all cases, all with $\mathrm{R} 0$ resection. Also in the series published by Coppa et al (27) there was no mortality, $40 \%$ morbidity, and an $83 \%$ overall 5 -year survival rate.

In the present case, we opted to attempt an IVC-preserving strategy using a modified hanging manoeuvre. In the original description by Belghiti et al (2) in 2001, a long vascular clamp was passed along the avascular plane at the 10-11 o'clock position on the anterior surface of the IVC in order to pass a tape to suspend the liver during transection. This could not be achieved for two reasons: i) Preoperative imaging demonstrated CRLM invasion into the anterolateral IVC over the 11 o'clock position and ii) the large, heavy CRLM exerted pres-

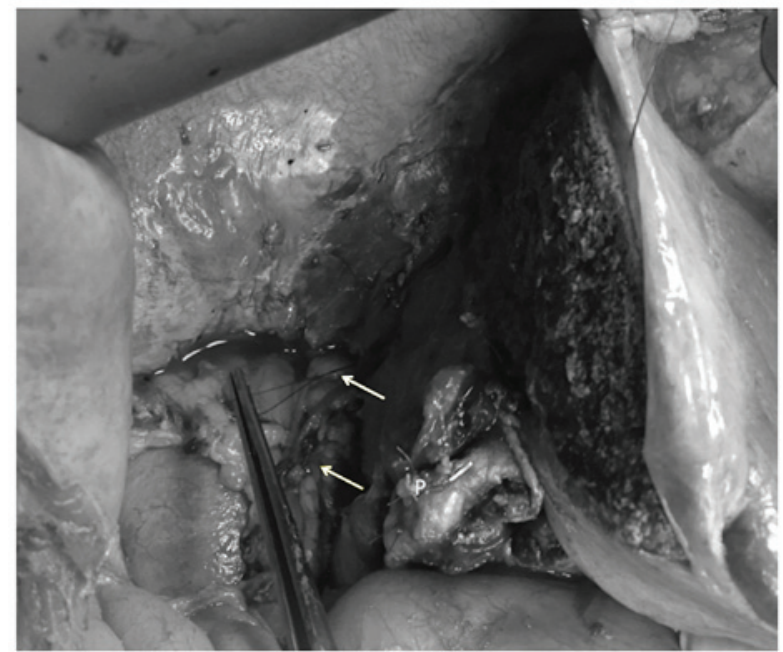

Figure 7. Extended right hepatectomy was completed, with no gross tumour in the liver remnant. The hepatic pedicle $(\mathrm{P})$ was preserved and the suture line used to reconstruct the inferior vena cava is seen (arrows).

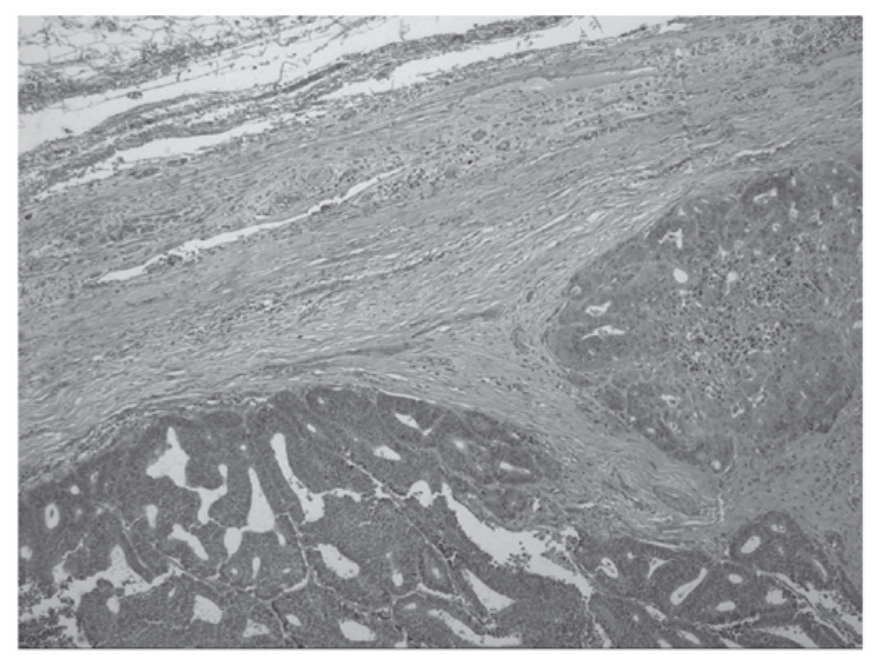

Figure 8. Low-power histological view of the inferior vena cava (IVC) with tumour deposits within its lumen and invasion of the IVC wall (hematoxylin and eosin staining; magnification, $\mathrm{x} 20$ ).

sure on the surface of the IVC, adding resistance to instrument passage. Thus, Belghiti's (2) classic hanging manoeuvre was modified by passing the instrument to the left of IVC at the 2-3 o'clock position. The instrument was directed cephalad to exit to the left of the left hepatic vein. Many caution against instrument passage at this location (25), as it is the usual location of the short hepatic and caudate veins. However, these are usually small veins, mostly sub-millimeter in diameter (32-36), and they vary in number, position and dimensions (33-35). If they are encountered during the hanging manoeuvre, the resultant bleeding is often mild due to the small diameter and low pressure within these veins. This bleeding should be easy to control by allowing the interrupted veins to be compressed by the weight of the liver. Moreover, Kanamura et al (37) demonstrated that similar short hepatic/caudate veins may be found in the classic avascular plane in $16.3 \%$ of the cases, and do not usually pose a problem. Some have described this manoeuvre under sonographic (38) or endoscopic $(39,40)$ guidance, in order to detect and avoid short 
hepatic veins when they exist; however, this was not available in our institution. In the present case, the encountered bleeding was minimal and easy to control with gentle, blind passage of the instruments aided by bimanual finger dissection, when necessary, as described by Aydin et al (41).

Once the plane was established, a 20Fr nasogastric tube was passed and used it to deliver traction anteriorly and to the left, in order to lift the liver and heavy CRLM, partially relieving the pressure applied onto the IVC. This in turn allowed passage of another instrument at the 1 o'clock position, with passing of a second tube directed between the middle and left hepatic veins. This double-tube hanging manoeuvre effectively lifted the central hepatic segments off the IVC, allowing us to safely define the intended resection plane. In 2008, Chen et al (42) described a double-tape hanging manoeuvre, where two tapes were placed in an avascular plane to the right of the IVC. The rationale for this modification was to achieve complete outflow control when the tapes were lifted in opposite directions (42). However, Liddo et al (25) pointed out that this manoeuvre could not guide transection, since the tapes were not positioned along the resection line. In our modification, the tubes were passed in different planes, the rationale being to safely identify the intended plane along the IVC that would be clear of tumour, in order to guide the transection lines. We acknowledge that this modification increases the risk of rupture of the short hepatic veins and, although we believe that the resultant bleeding would be mild and can be managed appropriately, we also acknowledge that this modification is unnecessary in simple resections; it only became necessary as we were unable to safely pass an instrument in the plane described by Belghiti et al (2).

Using these techniques followed by mobilization of the right liver allowed us to expose both sides of the IVC invaded by tumour and to pass a side-biting Satinsky clamp to allow controlled resection of the IVC wall to achieve clear margins. This selective IVC clamping technique was initially described by Togo et al (5) as a means to maintain flow through the systemic and hepatic circulations. It also allowed us to evaluate the degree of narrowing that resection and primary repair would yield, in order to decide whether total vascular exclusion and IVC replacement would be necessary.

Most authorities recommend IVC reconstruction, as complete ligation usually results in venous insufficiency and acute renal failure $(3,6,8)$. Ohwada et al $(7)$ recommended reconstruction with an interposed segment of polytetrafluoroethylene when primary closure would result in $>50 \%$ narrowing of the normal IVC diameter. In the present case, selective clamping revealed that resection would result in narrowing to $\sim 2 / 3$ of the original IVC diameter. Therefore, we opted for primary repair with vascular sutures, since this would sacrifice less caval wall compared with a stapled repair.

In conclusion, complex liver resection with IVC resection and reconstruction may be performed safely in centralized referral centers with dedicated hepatobiliary teams. Aggressive attempts at $\mathrm{R} 0$ resections are justified, since they are potentially curative for patients with CRLM. In these cases, hepatobiliary surgeons must be familiar with modified resection techniques, such as the classic hanging manoeuvre, anterior transection, selective caval clamping, total extravascular control, and IVC replacement/reconstruction. We recommend that this modification of the hanging manoeuvre be added to the armamentarium of hepatobiliary surgeons to improve safety in complex resections.

\section{References}

1. Fong Y, Gonen M, Rubin D, Radzyner M and Brennan MF: Long term survival is superior after resection for cancer in high volume centres. Ann Surg 242: 540-547, 2005.

2. Belghiti J, Guevara OA, Noun R, Saldinger PF and Kianmanesh R: Liver hanging maneuver: A safe approach to right hepatectomy without liver mobilization. J Am Coll Surg 193: 109-111, 2001.

3. Okada Y, Nagino M, Kamiya J, Yamamoto H, Hayakawa N and Nimura Y: Diagnosis and treatment of inferior vena caval invasion by hepatic cancer. World J Surg 27: 689-694, 2003.

4. Maeba T, Okano K, Mori S, Karasawa Y, Goda F, Wakabayashi H, Usuki $\mathrm{H}$ and Maeta $\mathrm{H}$ : Extent of pathologic invasion of the inferior vena cava in resected liver cancer compared with possible caval invasion diagnosed by preoperative images. J Hepatobiliary Pancreat Surg 7: 299-305, 2000.

5. Togo S, Shimada H, Tanaka K, Masui H, Fujii S, Endo I and Sekido $\mathrm{H}$ : Management of malignant tumor with intracaval extension by selective clamping of IVC. Hepatogastroenterology 43: 1165-1171, 1996.

6. Huguet C, Ferri M and Gavelli A: Resection of the suprarenal inferior vena cava. The role of prosthetic replacement. Arch Surg 130: 793-798, 1995.

7. Ohwada S, Ogawa T, Kawashima Y, Ohya T, Kobayashi I, Tomizawa N, Otaki A, Takeyoshi I, Nakamura S and Morishita Y: Concomitant major hepatectomy and inferior vena cava reconstruction. J Am Coll Surg 188: 63-71, 1999.

8. Duckett JW Jr, Lifland JH and Peters PC: Resection of the inferior vena cava for adjacent malignant diseases. Surg Gynecol Obstet 136: 711-716, 1973.

9. Schwartz SI: Right Hepatic Lobectomy. Am J Surg 148: 668-673, 1984.

10. Ozawa K: Hepatic function and liver resection. J Gastroenterol Hepatol 5: 296-309, 1990.

11. Liu CL, Fan ST, Lo CM, Tung-Ping Poon R and Wong J: Anterior approach for major right hepatic resection for large hepatocellular carcinoma. Ann Surg 232: 25-31, 2000.

12. Lai EC, Fan ST, Lo CM, Chu KM and Liu CL: Anterior approach for difficult major right hepatectomy. World J Surg 20: 314-318, 1996.

13. Liu CL, Fan ST, Cheung ST, Lo CM, Ng IO and Wong J: Anterior approach versus conventional approach right hepatic resection for large hepatocellular carcinoma: A prospective randomized controlled study. Ann Surg 244: 194-203, 2006.

14. Azoulay D, Marin-Hargreaves G, Castaing D, Adam R, Savier E and Bismuth $\mathrm{H}$ : The anterior approach: The right way for right massive hepatectomy. J Am Coll Surg 192: 412-417, 2001.

15. Abdalla EK, Noun R and Belghiti J: Hepatic vascular occlusion: Which technique? Surg Clin North Am 84: 563-585, 2004.

16. Li L, Wang HQ, Wang Q, Yang J and Yang JY: Anterior vs. conventional approach hepatectomy for large liver cancer: A meta-analysis. World J Gastroenterol 20: 17235-17243, 2014.

17. Miyazono F, Takao S, Natsugoe S, Uchikura K, Kijima F, Aridome K, Shinchi H and Aikou T: Molecular detection of circulating cancer cells during surgery in patients with biliary-pancreatic cancer. Am J Surg 177: 475-479, 1999.

18. Hayashi N, Egami H, Kai M, Kurusu Y, Takano S and Ogawa M: No-touch isolation technique reduces intraoperative shedding of tumor cells into the portal vein during resection of colorectal cancer. Surgery 125: 369-374, 1999.

19. Louha M, Nicolet J, Zylberberg H, Sabile A, Vons C, Vona G, Poussin K, Tournebize M, Capron F, Pol S, et al: Liver resection and needle liver biopsy cause hematogenous dissemination of liver cells. Hepatology 29: 879-882, 1999.

20. Capussotti L, Ferrero A, Russolillo N, Langella S, Lo Tesoriere R and Viganò L: Routine anterior approach during right hepatectomy: Results of a prospective randomised controlled trial. J Gastrointest Surg 16: 1324-1332, 2012.

21. Li SQ, Liang LJ, Peng BG, Yin XY, Lü MD, Kuang M, Li DM and Fu SJ: A comparative study of anterior versus conventional approach right hepatectomy for large hepatocellular carcinoma. Zhonghua Yi Xue Za Zhi 90: 1670-1673, 2010.

22. Wu TJ, Wang F, Lin YS, Chan KM, Yu MC and Lee WC: Right hepatectomy by the anterior method with liver hanging versus conventional approach for large hepatocellular carcinomas. Br J Surg 97: 1070-1078, 2010 . 
23. Takács I, Furka A, Kotán R, Boland Mehrdad G, Pósán J, Vágvölgyi A, Hallay J and Sápy P: Anterior approach for liver resection in the cases of the treatment of large liver tumors. Magy Seb 59: 362-368, 2006.

24. Wang CC, Jawade K, Yap AQ, Concejero AM, Lin CY and Chen CL: Resection of large hepatocellular carcinoma using the combination of liver hanging maneuver and anterior approach. World J Surg 34: 1874-1878, 2010.

25. Liddo G, Buc E, Nagarajan G, Hidaka M, Dokmak S and Belghiti J: The liver hanging manoeuvre. HPB 11: 296-305, 2009.

26. Llado L, Muñoz A, Ramos E, Torras J, Fabregat J and Rafecas A The anterior hanging-approach improves postoperative course after right hepatectomy in patients with colorectal liver metaseases. Resutls of a prospective study with propensity-score mathing comparison. Eur J Surg Oncol 42: 176-183, 2016.

27. Coppa J, Citterio D, Cotsoglou C, Germini A, Piccioni F, Sposito C and Mazzaferro V: Transhepatic anterior approach to the inferior vena cava in large retroperitoneal tumours resected en bloc with the right liver lobe. Surgery 154: 1061-1068, 2013.

28. Beppu T, Ishiko T, Chikamoto A, Komori H, Masuda T, Hayashi H, Okabe H, Otao R, Sugiyama S, Nasu J, et al: Liver hanging maneuver decreases blood loss and operative time in a right side hepatectomy. Hepatogastroenterology 59: 542-545, 2012 .

29. Hwang S, Lee SG, Yee YJ, Kim KH, Ahn CS, Kim KW, Ko KH and Choi NK: Modified liver hanging maneuver to facilitate left hepatectomy and caudate lobe resection for hilar bile duct cancer. J Gastrointest Surg 12: 1288-1292, 2008.

30. Nanashima A, Sumida Y, Abo T, Takeshita H, Hidaka S, Sawai T, Yasutake $\mathrm{T}$ and Nagayasu T: Trisectionectomy for large hepatocellular carcinoma using the liver hanging maneuver. Eur J Surg Oncol 35: 326-330, 2009.

31. Cawich SO, Thomas DA, Ramjit C, Bhagan R and Naraynsingh V: Complex liver resections for colorectal metastases: Are they safe in a low-volume, resource-poor caribbean setting? Case Rep Surg 2015: 570968, 2015

32. Kogure K, Kuwano H, Fujimaki N and Makuuchi M: Relation among portal segmentation, proper hepatic vein, and external notch of the caudate lobe in the human liver. Ann Surg 231: 223-228, 2000.

33. Hirai I, Murakami G, Kimura W, Kanamura T and Sato I: How should we treat short hepatic veins and paracaval branches in anterior hepatectomy using the hanging manoeuvre without mobilization of the liver? An anatomical and experimental study. Clin Anat 16: 224-232, 2003.

34. Sato TJ, Hirai I, Murakami G, Kanamura T, Hata F and Hirata K: An anatomical study of short hepatic veins, with special reference to delineation of the caudate lobe for hanging manoeuvre of the liver without the usual mobilization. J Hepatobiliary Pancreat Surg 9: 55-60, 2002.
35. Trotovsek B, Belghiti J, Gadzijev EM, Ravnik D and Hribernik M: Anatomical basis of the liver hanging manoeuvre. Hepatogastroenterology 52: 728-730, 2005.

36. Kogure K, Kuwano H, Yorifuji H, Ishikawa H, Takata K and Makuuchi M: The caudate processus hepatic vein: A boundary hepatic vein between the caudate lobe and the right liver. Ann Surg 247: 288-293, 2008.

37. Kanamura T, Murakami G, Hirai L, Hata F, Sato TJ, Kumon M and Nakajima Y: High dorsal drainage routes of Spiegel's lobe. J Hepatobiliary Pancreat Surg 8: 549-556, 2001.

38. Kokudo N, Imamura H, Sano K, Zhang K, Hasegawa K, Sugawara Y and Makuuchi M: Ultrasonically assisted retrohepatic dissection for a liver hanging manoeuvre. Ann Surg 242: 651-654, 2005.

39. Lai PB, Wong J, Ng WW, Lee WL, Cheung YS, Tsang YY and Lee KF: Safe modification of the liver-hanging manoeuvre by endoscopic-assisted dissection of the retrohepatic tunnel. Surg Today 37: 915-917, 2007.

40. Meng WC, Shao CX, Mak KL, Lau PY, Yeung YP and Yip AW: Anatomical justification of Belghiti's 'liver hanging manoeuvre' in right hepatectomy with anterior approach. ANZ J Surg 73 407-409, 2003.

41. Aydin U, Yazici P, Zeytunlu M, Kilic M and Coker A: Bimanual 'bi-finger' liver hanging manoeuvre: An alternative and safe technique for liver hanging. HPB (Oxford) 9: 195-198, 2007.

42. Chen XP, Zhang WG, Lau WY and Qiu FZ: Right hepatectomy using the liver double-hanging manoeuvre through the retrohepatic avascular tunnel on the right of the inferior vena cava. Surgery 144: 830-833, 2008. 